\title{
A Possible Explanation for the Acceleration of the Universe's Expansion without Dark Energy
}

\author{
Dongbiao Kang \\ School of Physics and Electrical Engineering, Anyang Normal University, Anyang, China \\ Email: dbkang@aynu.edu.cn
}

How to cite this paper: Kang, D.B. (2021) A Possible Explanation for the Acceleration of the Universe's Expansion without Dark Energy. Journal of Modern Physics, 12, 594-604.

https://doi.org/10.4236/jmp.2021.125038

Received: March 9, 2021

Accepted: April 13, 2021

Published: April 16, 2021

Copyright $\odot 2021$ by author(s) and Scientific Research Publishing Inc. This work is licensed under the Creative Commons Attribution International License (CC BY 4.0).

http://creativecommons.org/licenses/by/4.0/

(c) (i) Open Access

\begin{abstract}
The acceleration of the expansion of the universe has been observed. To explain this phenomenon, physicists usually introduce the dark energy (DE) whose pressure is negative, or they need to modify the gravity to produce a term equivalent to the dark energy. Are there other possibilities? Combining our previous works about statistical mechanics of self-gravitating system with the derivation of van der waals equation, we propose a different matter's equation of state (EoS) in this paper. Then, we find that if the matter's density is low enough, its pressure can always be negative, which means that it is the matter that drives the expansion's acceleration. So here we will not need to add the DE to the universe. Our results also predict that the universe finally will tend to be dominated by an approximately constant energy density whose value can be smaller than $\mathrm{DE}$. The data of Supernova cannot differentiate our model from the standard model, but they may possibly indicate some deviations from $\Lambda \mathrm{CDM}$.
\end{abstract}

\section{Keywords}

Cosmology, Statistical Mechanics, Dark Energy, Dark Matter

\section{Introduction}

Under the assumption of isotropy and homogeneity of our Universe at large scale structure, people make a successful explanation to the cosmology accelerating expansion [1] by introducing a new component called DE, which constitutes of about $70 \%$ energy of the universe. Up to now, the most successful view about the $\mathrm{DE}$ is the $\Lambda \mathrm{CDM}$ model in which the energy density of $\mathrm{DE}$ is constant. Although $\Lambda \mathrm{CDM}$ model is very consistent with all observational data, it faces the fine tuning problem [2] and coincidence problem. Alternatively, plenty of other DE models have also been proposed ([3] [4] [5] [6], also see some reviews, such 
as [7] [8]), but almost all of them solves the acceleration problem either by introducing new degree of freedom or by modifying gravity, which challenges both cosmology and nuclear physics.

However, do we really need the DE? Or do we need so much DE? We notice that commonly the matter's EoS comes from the classical thermodynamics without consideration of the gravity, has it been confirmed to be reasonable at the cosmological scale? We think that the answer may be not sure, and [9] has ever considered the dark matter as van der waals gas to explain the acceleration of the universe's expansion. Besides, our latest studies also provide different understandings of the long-range statistical mechanics as simply shown in the following: if we apply the Boltzmann entropy

$$
S=-\int f \ln f \mathrm{~d} \tau
$$

into the Newtonian self-gravitating system, with the principle of maximum entropy we will always obtain the isothermal solution with infinite mass and energy [10], which is a serious problem; [11] preliminarily studies the entropy form taken by [12] and proposes that a self-gravitating system's entropy may be a saddle point and not a maximum; [13] completes the variation process of entropy and confirms that we can obtain an EoS which is different from the isothermal solution and can give finite mass; [14] shows the different thermodynamics of self-gravitating system.

In this paper, we will further discuss that the general relativity may also have similar effects on the matter's EoS at the cosmological scale. In the next section, we will show our findings and propose a reason for the universe's accelerating expansion without DE. In section 3, we will use the data of supernova to shorten the range of the values of the parameters in our model. Some discussions and conclusions will be made in the final section. In this paper, we set $c=1$ and use "0" to denote quantity's current value except $\Omega_{x 0}$ below.

\section{Explanation for the Acceleration}

According to the cosmological principle, the universe is homogeneous and isotropic. The homogeneity and isotropy of the universe requires the energy-momentum tensor of the matter to be written as

$$
T^{\mu v}=(\rho+p) U^{\mu} U^{v}+p g^{\mu v} .
$$

where $\rho$ is the energy density, $p$ is the pressure, and $U^{\mu}$ is the velocity four-vector. After we substitute (2) into the Einstein's equation, we can get the Friedmann equation:

$$
\ddot{a}=-4 \pi G\left(\frac{\rho}{3}+p\right) a, \dot{a}^{2}+\kappa=\frac{8 \pi G}{3} \rho a^{2},
$$

where $a$ is the scale factor. The energy-momentum conservation law is

$$
\dot{\rho}=-\frac{3 \dot{a}}{a}(\rho+p),
$$


which also can be obtained from Equation (3). Combining the EoS $p=p(\rho)$ with Equation (3), we can get the solution of $a, p$ and $\rho$. After the inflation, the universe is assumed to be a gas whose pressure is [15]

$$
p=\frac{v^{2}}{3} \rho,
$$

where $v^{2}$ is the mean-square velocity, $m$ is the mass of particle, and $T$ is the defined temperature. We usually use $p=0$ and $p=\rho / 3$ to denote the matter $\left(v^{2}=\frac{3 k T}{m} \ll 1\right)$ and radiation's $(v=1)$ EoSs respectively, but by Equation (5) we cannot understand the nature of the $\mathrm{DE}$ whose pressure is negative, even commonly we understand DE by that it does negative work to the universe.

Notice that Equation (5) can be derived by two ways [16]: we can use the related formulas of the classical statistical mechanics to calculate the matter and radiation's EoSs, both of which satisfy Equation (5); it also can be obtained by the kinetics alone, which needs to consider the bombardment by the particles (rigid body) on the walls of the container to calculate the kinetic pressure. However, the classical statistical mechanics does not consider the effects of gravity; besides, the wall of the container does not exist in the universe, and in fact we can treat the wall to be a potential well with minus infinity at the boundary for the particles, while the gas in the universe is in the gravitational potential well which is different from the wall. If we treat the universe as a thermodynamical system whose particles interact by gravity, will these two ways mentioned above still be available under the cosmic background where the effect of general relativity cannot be neglected? We think that the answer is yes for the radiation, because the effect of gravity can be neglected compared with the electromagnetic force; but for the matter whose main component is the dark matter for which the gravity plays the most important role, the answer may be not sure. In [13] by the method of statistical mechanics we have obtained an EoS which is used to describe the thermodynamical equilibrium state of a virialized Newtonian self-gravitating system:

$$
\rho=\beta p+\alpha p^{4 / 5},
$$

where $\beta$ and $\alpha$ are positive and determined by the energy and mass respectively. $\beta$ has been identified to $m / k T$ in [14]. Notice that the power index of the second term of Equation (6)'s right ride is less than 5/6, which ensures that the mass and energy are finite galdyn08. From Equation (6) we can obtain $p=p(\rho)$, which can be approximately written as [14]

$$
p=\frac{k T \rho}{m}-\alpha\left(\frac{k T}{m}\right)^{\frac{9}{5}} \rho^{\frac{4}{5}} .
$$

Of course, Equation (7) is different from Equation (5) even we let $v^{2}=3 k T / m$. Next we will compare Equation (6) with the van der waals to further explain the possible reasonability of Equation (6). The van der waals equation is: 


$$
\left(p+\frac{n^{2} a}{V^{2}}\right)(V-n b)=N k T
$$

where $a$ and $b$ is used to describe the attractive and repulsive interactions among the molecules of gas respectively, and they are determined by the potential of the molecules' two-body interactions and can be calculated by the method of cluster expansion in the canonical ensemble (see some books of statistical mechanics such as [16]). If the potential of the molecules' two-body interactions has other forms, $a$ and $b$ will still appear in Equation (8) but their values will be changed. When we neglect the repulsive interactions and set $b=0, V=N m / \rho$, Equation (8) becomes

$$
p=\frac{k T}{m} \rho-a\left(\frac{n}{N m}\right)^{2} \rho^{2}
$$

which is very analogous to Equation (7), even we can speculate that existence of $\alpha$ in Equation (7) should be a requirement of possible statistical mechanics dealing with Newtonian gravitating systems. This speculation is also supported by the following: the two-body interaction is the main interaction in van der waals gas and $a$ and $b$ in Equation (8) are related to the two-body interaction; while the force on the single particle of gravitating system is mainly determined by the gravitational field generated by all the particles [10], and coincidentally $\alpha$ in Equation (7) is determined by the total mass. The change of index from 2 to 4/5 may be caused by the change from two-body interactions among molecules to long-range Newtonian gravity. If this speculation is correct, can general relativity cause similar effect on the EoS of the matter? We do not know the answer, but if such theory exists we may do some guess of its results as the following: based on above analysis, we find that $\beta$ is related to the temperature, $\alpha$ is necessary to denote the effect of the long-range statistical mechanics, and neither of them will disappear if the gravity changes from the Newtonian to the general relativity; but the index 4/5 in Equation (7) may be changed in the general relativity. So we assume that at the cosmological scale the EoS of the matter may be written as

$$
p_{m}=\frac{v^{2}}{3} \rho_{m}-\Omega_{x 0} \rho_{0}^{1-t} \rho_{m}^{t}, t<1, \Omega_{x 0}>0
$$

where $\rho_{0}=\frac{3 H_{0}^{2}}{8 \pi G}, t$ is a constant, and $v$ and $\Omega_{x 0}$ are about constants or very slowly changing functions of the time at the matter dominated era. Here we temporally do not assume that $v^{2}$ is as small as its value in $\Lambda$ CDM. Notice that the form of Equation (10) can be directly borrowed from the van der waals equation, and only $t<1$ is speculated from our previous works. Notice that [9] even considers the dark matter as the van der waals gas with an EoS $p=\gamma \rho /(1-\beta)-\alpha \rho^{2}$ to explain the acceleration problem, which is very similar to this paper. Besides, [17] treats the dark matter interaction as an alternative of dark energy; [18] [19] also introduce the fluids with $p=-\rho-A \rho^{\alpha}$, which is to 
describe the dark energy. These works are similar but different from this paper.

Now we will analyze the effect of Equation (10) on the universe's evolution. Our model only modifies the pressure of matter, so the cosmic history before the matter-dominated era is almost not effected and we only consider the later time. From observations we know that the universe is always expanding, so $\rho_{m}$ is decreasing with time. When $\rho_{m}$ is large enough, $p_{m}$ is positive but is smaller than Equation (5). After some time, there will be an era described by $p_{m} \sim 0$, just like CDM. But it is evident that the pressure in Equation (10) can be negative if

$$
\left(\frac{\rho_{m}}{\rho_{0}}\right)^{1-t}<\frac{\Omega_{x 0}}{v^{2}},
$$

which tells us that when the matter reaches the thermodynamical equilibrium, its pressure will be negative if its density is low enough. This really is a surprising thing but may be natural in our model. Here we will also mention the work of [20] which proves that the N-particle system's thermodynamic pressure cannot be negative if the force between two particles is short range, while coincidentally the gravity is long-range, which means that the pressure of $\mathrm{N}$-particle gravitating system has not been ensured to be not negative. Because the matter's density is always decreasing, it will satisfy Equation (11) at some time, then can this negative pressure accelerate the universe's expansion? This requires $p<-\rho / 3$, i.e.

$$
\left(\frac{\rho_{m}}{\rho_{0}}\right)^{1-t}<\frac{3 \Omega_{x 0}}{3 v^{2}+1} .
$$

Of course this equation also can be satisfied because of the expansion of the universe, so we find that if the long-range statistical mechanics is really different from the classical one and can produce an EoS like Equation (10), it will be the matter that drives the universe's accelerated expansion! The next question is how the universe will evolve in the future, which requires some detailed calculations. We substitute Equation (10) into the Equation (4), then the matter's density will evolve as

$$
\frac{\rho_{m}}{\rho_{0}}=\left[\frac{C\left(\frac{a_{0}}{a}\right)^{3 B(1-t)}+\Omega_{x 0}}{B}\right]^{\frac{1}{1-t}}=\left[\left(\frac{C}{B}\right)^{1-t}\left(\frac{a_{0}}{a}\right)^{3 B}+\cdots+\left(\frac{\Omega_{x 0}}{B}\right)^{\frac{1}{1-t}}\right]
$$

where

$$
B=1+\frac{v^{2}}{3}, C=B\left(1-\Omega_{r 0}\right)^{1-t}-\Omega_{x 0},
$$

and $\Omega_{r}$ is the current radiation's density parameter. Notice that $1<B<4 / 3$. From observations we know $\kappa \sim 0$ and $\Omega_{r 0} \sim 0.04$, if we define

$$
\Omega_{m}=\frac{\rho_{m}}{\rho_{0}}
$$


its current value $\Omega_{m 0}=1-\Omega_{r 0}$ will be much closer to 1 , which is a major difference of our model from $\Lambda$ CDM. When $\Omega_{x 0}=0$ (or $a$ is small enough so that $\Omega_{x 0}$ can be neglected) and $B$ tends to be 1, Equation (13) will become $\rho \propto a^{-3}$. If $B=1$ and $t=0$, the form of Equation (13) will be the same as a direct addition of the matter and DE, and our model gives the same results as $\Lambda \mathrm{CDM}$, but even at this case our results still provide a different understanding of the universe, and any deviations from $B=1$ or $t=0$ will indicate the preferences of our model. Because of the universe's expansion, from Equation (13) we can easily find that the density will finally approximately become a constant

$$
\rho_{x}=\rho_{0}\left(\frac{\Omega_{x 0}}{B}\right)^{\frac{1}{1-t}},
$$

If we neglect the variations of $v$ and $\Omega_{x 0}$ with time. So our model predicts almost the same destiny of the universe as $\Lambda \mathrm{CDM}$ : the standard model says that the energy consists of the radiation, the matter and the DE, with the expansion of the universe the DE will dominate and the expansion will accelerate; while our result states that because there is some unknown physics such as long-range statistical mechanics, even the universe always expands there is a no-zero minimum of the energy density of the matter, which also accelerates the expansion of the universe. However, this minimized density can be smaller than DE because: from Equation (3) we know that $\ddot{a}>0$ requires that $\rho$ decreases slower than $a^{-2}$, so from Equation (13) we know that not only the constant term but also other terms whose power indexes are larger than -2 makes the expansion accelerate. So the value of Equation (16) should be smaller than DE if $t>0$. If in the future the universe's density becomes a constant, according to Equation (3) our results may give a smaller value of the Hubble parameter than the standard model, which also differentiates our results from the standard model.

\section{Compared with the Data of SNIa}

\subsection{Data Analysis}

Next we will analyze the data of SNIa. First we need to calculate the Hubble parameter. It can be expressed by four parameters $\left(t, \Omega_{x 0}, B, h_{0}\right)$ in our model,

$$
\frac{H(z)}{H_{0}}=\left(\frac{C(1+z)^{-3 B(1-t)}+\Omega_{x 0}}{B}\right)^{\frac{1}{1-t}}+\Omega_{r 0}(1+z)^{4} .
$$

where $h_{0}$ is the present value of the Hubble parameter $H_{0}$ in unit $100 \mathrm{~km} / \mathrm{s} / \mathrm{Mpc}$ and $z$ is the redshift.

To check our proposals made in Sec. II and to constrain the model parameters, we take use of the Union2 dataset [21] and the Hubble evolution data. The Union2 dataset contains 557 type Ia SN data and covers the redshift range $z=[0.015,1.4]$, including samples from other surveys, such as CfA3 [22], SDSS-II Supernova Search [23] and high-z Hubble Space Telescope.

We fit the SNIa data by minimizing the $\chi^{2}$ value of the distance modulus. 
$\chi_{s n}^{2}$ for SNIa is obtained by comparing theoretical distance modulus $\mu_{t h}(z)=5 \log _{10}\left[(1+z) \int_{0}^{z} \mathrm{~d} x / E(x)\right]+\mu_{0}$

$\left(\mu_{0}=42.384-5 \log _{10} h_{0}, E(z)=H(z) / H_{z}\right)$ with observed $\mu_{o b}$ of supernova:

$$
\chi_{s n}^{2}=\sum_{i}^{557} \frac{\left[\mu_{t h}\left(z_{i}\right)-\mu_{o b}\left(z_{i}\right)\right]^{2}}{\sigma^{2}\left(z_{i}\right)} .
$$

To reduce the effect of $\mu_{0}$, we expand $\chi_{s n}^{2}$ with respect to $\mu_{0}$ [24]:

$$
\chi_{s n}^{2}=F+2 G \mu_{0}+H \mu_{0}^{2}
$$

where

$$
\begin{gathered}
F=\sum_{i} \frac{\left[\mu_{t h}\left(z_{i} ; \mu_{0}=0\right)-\mu_{o b}\left(z_{i}\right)\right]^{2}}{\sigma^{2}\left(z_{i}\right)}, \\
G=\sum_{i} \frac{\mu_{t h}\left(z_{i} ; \mu_{0}=0\right)-\mu_{o b}\left(z_{i}\right)}{\sigma^{2}\left(z_{i}\right)} \\
H=\sum_{i} \frac{1}{\sigma^{2}\left(z_{i}\right)}
\end{gathered}
$$

(18) has a minimum as

$$
\tilde{\chi}_{s n}^{2}=\chi_{s n, \min }^{2}=F-G^{2} / H
$$

which is independent of $\mu_{0}$. In fact, it is equivalent to perform an uniform marginalization over $\mu_{0}$, and the difference between $\tilde{\chi}_{s n}^{2}$ and the marginalized $\chi_{s n}^{2}$ is just a constant [17]. We will adopt $\tilde{\chi}_{s n}^{2}$ as the goodness of fit between theoretical model and SNIa data.

We also use the 12 Hubble evolution data from [25] and [26], its $\chi_{H}^{2}$ is defined as

$$
\chi_{H}^{2}=\sum_{i=1}^{12} \frac{\left[H\left(z_{i}\right)-H_{o b}\left(z_{i}\right)\right]^{2}}{\sigma_{i}^{2}} .
$$

Note that the redshift of these data falls in the region $z \in(0,1.75)$.

In summary,

$$
\chi_{t o t}^{2}=\tilde{\chi}_{s n}^{2} \chi_{H}^{2}
$$

and we assume uniform priors on all the parameters. We also prior assume that the age of our universe $T_{0}$ satisfies $10 \mathrm{Gyr}<T_{0}<20 \mathrm{Gyr}$.

\subsection{Results and Discussions}

The analysis is performed by using the Monte Carlo Markov Chain in the multidimensional parameter space to derive the likelihood. Naturally, we employ some physically obvious limitations to make the estimation of parameters more robust, for example, we set $t<1,1 \leq B<4 / 3$ in our analysis. We first investigate the constraint on the model parameters, and the best-fit values and errors of parameters are summary in Table 1 . We also plot the $1 \mathrm{D}$ marginalized distribution probability of each parameter, shown in Figure 1. The likelihood distribution 
shows a remarkable deviation from the Gaussian distribution, which result in a discrepancy between the best-fit values and the expected values of the parameters, as consistent with the result in Table 1.

Further, we focus on parameters $(t, B)$, which play a more important role in our model. The 2D contour plot is shown in Figure 2, which shows that the results of standard $\Lambda \mathrm{CDM}$ model are contained in our model. The $2 \sigma$ errors mean that the case with $t>0.80$ is excluded at $95.4 \%$ confidence level, which is very consistent with our speculation. But constraint on $B$ cannot be obtained.
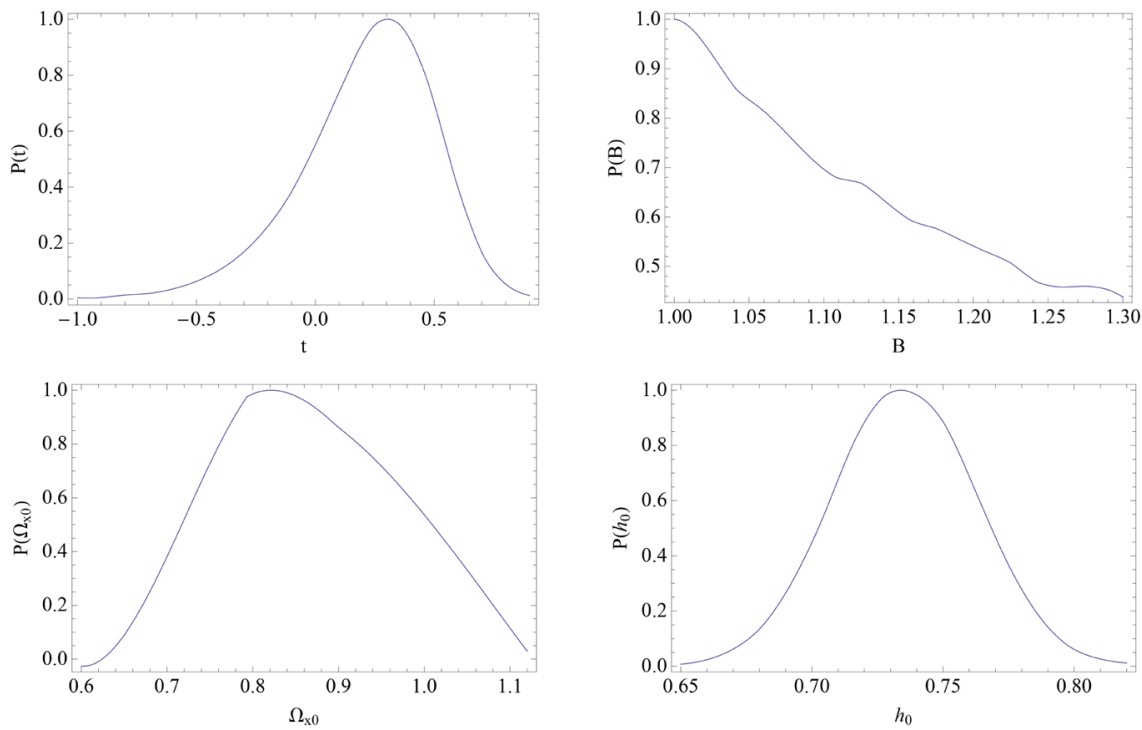

Figure 1. 1D marginalized distribution probability of $t, \Omega_{x 0}, B, h_{0}$.

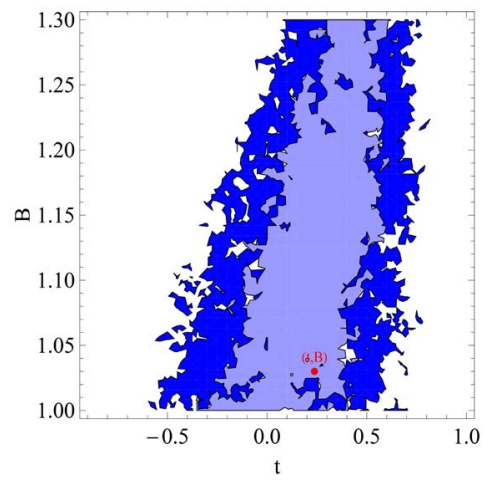

Figure 2. 68\% and $95 \%$ contour plot in $t-B$ plane.

Red dot in the center is the expected value.

Table 1. Expected values, $1-\sigma$ and $2-\sigma$ error of $t, \Omega_{x 0}, B, h_{0}$ in this model. The second line is in form of Expectation ${ }_{+1 \sigma,+2 \sigma}^{-1 \sigma,-2 \sigma}$. And the best-fit values are also shown in the last line, which are different from the expected values.

\begin{tabular}{cccc}
\hline$t$ & $\Omega_{x 0}$ & $B$ & $h_{0}$ \\
\hline $0.237_{+0.346,+0.562}^{-0.347,-1.237}$ & $0.824_{+0.200,+0.271}^{-0.107,-0.161}$ & 1.030 & $0.724_{+0.055,+0.074}^{-0.025,-0.022}$ \\
0.051 & 0.734 & 1.008 & 0.738
\end{tabular}


From above results, we find that the data of SNIa cannot differentiate our model from $\Lambda \mathrm{CDM}$. We need to consider the perturbation theory to further test our model, which will be another work. But from Table 1 and Figure 1 we can see that the data seem to indicate some deviations from $B=1$ and $t=0$, i.e. $\Lambda \mathrm{CDM}$. Notice that according to current criteria, the dark matter is called to be cold if $v<0.1^{1}$, which means that $B<1.0033$, so there is only a very short range of $B$ for the cold matter. However, both the best-fit and expected value of $B$ are larger than 1.0033 , which suggests that the matter may be not so cold as $\Lambda \mathrm{CDM}$ predicts. This conclusion also agrees with some works [27] [28] which aim to use the warm dark matter to solve the contradictions between observations and numerical simulations of $\Lambda \mathrm{CDM}$ at the galaxy scale.

\section{Conclusions}

Our previous works have proved that the thermodynamics of gravitating systems at the galaxy scale may be different from the classical one, and we obtain an EoS like Equation (7), which is in analogy with the van der waals equation. We speculate that it is the long-range statistical mechanics that produces the Equation (7). Based on possible effect of the general relativity on the matter's EoS, we propose that at the cosmological scale, the form of the matter's EoS may be like the Equation (10). Then, we find that with the universe's expansion the matter's density will decrease with time to be a no-zero minimized constant, and it is the matter that drives the expansion's acceleration. Our work is similar to [9], but with different form of EoS and different motivation. So our model may provide a new kind of explanation for the expansion's acceleration, and there are three major different results of our model from $\Lambda \mathrm{CDM}$ :

1). our new defined $\Omega_{m}=1-\Omega_{r}$ is close to 1 and is much larger than its value in $\Lambda \mathrm{CDM}$;

2). $B$ and $t$ may be not exactly to 1 and 0 respectively;

3). if the universe's density becomes a constant in the future, its value can be smaller than DE.

It is easier to compare our model with $\Lambda \mathrm{CDM}$ by the second point, so we use the data of SNIa to constrain the value of $B$ and $t$. After analyzing the data's, we cannot differentiate our model from $\Lambda \mathrm{CDM}$, but there seems to be some indications of deviations from $\Lambda \mathrm{CDM}$. To further test our model, we need to consider the density's perturbation in the future.

\section{Acknowledgements}

DBK is very grateful for Zhong-Liang Tuo's many helps of dealing with the data of supernova. This work is supported by the National Natural Science Foundation of China, No. 11947098.

\section{Conflicts of Interest}

The author declares no conflicts of interest regarding the publication of this paper. hhttp://en.wikipedia.org/wiki/Dark_matter. 


\section{References}

[1] Perlmutter, S., et al., Supernova Cosmology Project Collaboration (1999) The Astrophysical Journal, 517, 565. arXiv:astro-ph/9812133

[2] Copeland, E.J., Sami, M. and Tsujikawa, S. (2006) International Journal of Modern Physics D, 15, 1753.

[3] Copeland, E.J., Sami, M. and Tsujikawa, S. (2006) International Journal of Modern Physics D, 15, 1753. https://doi.org/10.1142/S021827180600942X

[4] Caldwell, R.R. and Steinhardt, P.J. (1998) Physical Review D, 57, 6057. https://doi.org/10.1103/PhysRevD.57.6057

[5] Steinhardt, P.J., Wang, L.M. and Zlatev, I. (1999) Physical Review D, 59, Article ID: 123504. https://doi.org/10.1103/PhysRevD.59.123504

[6] Capozziello, S., Cardone, V.F., Carloni, S. and Troisi, A. (2003) International Journal of Modern Physics D, 12, 1969.

[7] Nojiri, S.I. and Odintsov, S.D. (2011) Physics Reports, 505, 59. https://doi.org/10.1016/j.physrep.2011.04.001

[8] Cai, Y.-F., Saridakis, E.N., Setare, M.R. and Xia, J.-Q. (2010) Physics Reports, 493, 1. https://doi.org/10.1016/j.physrep.2010.04.001

[9] Capozziello, S., Cardone, V.F., Carloni, S., De Martino, S., Falanga, M., Troisi, A. and Bruni, M. (2005) JCAP, 0504, 005. https://doi.org/10.1088/1475-7516/2005/04/005

[10] Binney, J. and Tremaine, S. (2008) Galactic Dynamics. 2nd Edition, Princeton University Press, Princeton. https://doi.org/10.1515/9781400828722

[11] He, P. and Kang, D.B. (2010) MNRAS, 406, 2678. https://doi.org/10.1111/j.1365-2966.2010.16869.x

[12] White, S.D.M. and Narayan, R. (1987) MNRAS, 229, 103. https://doi.org/10.1093/mnras/229.1.103

[13] Kang, D.B. and He, P. (2011) MNRAS, 416, 32. https://doi.org/10.1016/j.destud.2011.05.002

[14] Kang, D.B. (2011) Different Thermodynamics of Self-Gravitating Systems and Discussions for Some Observations and Simulations.

[15] Liddle \& Lyth (2000) Cosmological Inflation and Large-Scale Structure. Cambridge University Press, Cambridge. https://doi.org/10.1017/CBO9781139175180

[16] Pathria, R.K. (1996) Statistical Mechanics. 2nd Edition, Butterworth-Heinemann, Oxford.

[17] Basilakos, S. and Plionis, M. (2009) $A \& A$, 507, 47B. https://doi.org/10.1051/0004-6361/200912661

[18] Stefancic, H. (2005) Physical Review D, 71, Article ID: 084024. https://doi.org/10.1103/PhysRevD.71.124036

[19] Nojiri, S.I. and Odintsov, S.D. (2005) Physical Review D, 72, Article ID: 023003. https://doi.org/10.1103/PhysRevD.72.023003

[20] van Hove, L. (1949) Physica, 15, 11. https://doi.org/10.1016/0031-8914(49)90059-2

[21] Amanullah, R., et al. (2010) The Astrophysical Journal, 716, 712.

[22] Hicken, M., et al. (2009) The Astrophysical Journal, 700, 1097.

[23] Holtzman, J.A., et al. (2008) The Astrophysical Journal, 136, 2306.

[24] Nesseris, S. and Perivolaropoulos, L. (2005) Physical Review D, 72, Article ID: 
123519. https://doi.org/10.1103/PhysRevD.72.123519

[25] Simon, J., Verde, L. and Jimenez, R. (2005) Physical Review D, 71, Article ID: 123001. https://doi.org/10.1103/PhysRevD.71.123001

[26] Gaztanaga, E., Cabre, A. and Hui, L. (2008) Clustering of Luminous Red Galaxies IV: Baryon Acoustic Peak in the Line-of-Sight Direction and a Direct Measurement of $\mathrm{H}(\mathrm{z})$.

[27] Colin, P., Avila-Reese, V. and Valenzuela, O. (2000) The Astrophysical Journal, 542, 622. https://doi.org/10.1086/317057

[28] Bode, P., Ostriker, J.P. and Turok, N. (2001) The Astrophysical Journal, 556, 93. https://doi.org/10.1086/321541 